\title{
Preservación de tradiciones en el ámbito culinario para fortalecer la sustentabilidad turística en Xicotepec mediante la capacitación académica
}

\section{Preservation of traditions in the culinary field to strengthen tourism sustainability in Xicotepec through academic training}

GONZÁLEZ DE LOS MONTERO-SIERRA, María Joséł*, CRUZ-CABRERA, Clotilde, VERGARA-HERRERA, Francisco Javier y CARMONA-GONZÁLEZ, Juan Carlos

\author{
Universidad Tecnológica de Xicotepec de Juárez. Av. Universidad Tecnológica No. 1000 Tierra Negra, 73080 Xicotepec de \\ Juárez, Pue.
}

ID $1^{\text {er }}$ Autor: María José, González De Los Montero-Sierra / ORC ID: 0000-0002-4124-2757, Researcher ID Thomson: Y-2510-2019, CVU CONACYT ID: 1005105

ID $1^{\text {er }}$ Coautor: Clotilde, Cruz-Cabrera / ORC ID: 0000-0002-4516-7061, Researcher ID Thomson: S-6899-2018, CVU CONACYT ID: 953328

ID $2^{\text {do }}$ Coautor: Francisco Javier, Vergara-Herrera / ORC ID: 0000-0003-4206-0362, Researcher ID Thomson: W26082019, CVU CONACYT ID: 1005090

ID $3^{\text {er }}$ Coautor: Juan Carlos, Carmona-González, / ORC ID: 0000-0002-2489-7882, Researcher ID Thomson: Y-18042018, CVU CONACYT ID: 954425

DOI: $10.35429 / J P D .2019 .9 .3 .8 .13$

Recibido 15 de Julio, 2019; Aceptado 28 de Septiembre, 2019

\section{Resumen}

El proyecto documenta la investigación realizada sobre los platillos tradicionales de Xicotepec, pues, aunque cuenta con la denominación de Pueblo Mágico y esto incentiva el turismo, poco es el impacto que ha tenido para el sector gastronómico, siendo atractivo una gastronomía única para los que buscan conocer a México a través de sus alimentos. Una cocina que, por la constante migración y globalización, poco a poco se va perdiendo, para ello se encuestaron a entidades económicas y a habitantes de diferentes lugares que preparan alimentos (700 la muestra), con visitas y prácticas con las cocineras tradicionales de cada comunidad. Con base en el análisis e interpretación de la información se obtuvieron resultados favorables con respecto a la cocina, sus ingredientes y formas de preparación, y se elaboró un recetario anecdótico con la finalidad de capacitar a los estudiantes de gastronomía de la Universidad Tecnológica de Xicotepec de Juárez, estudiantes de bachillerato con capacitación en Alimentos y a alumnos del Centro de Capacitación y Desarrollo (CECADE), con el fin de promover la preservación culinaria y el interés en la investigación gastronómica local para lograr una especialización y mejorar la elaboración de los platillos típicos de la región en sus futuros centros de trabajo.

Turismo, Gastronomía, Preservación

\begin{abstract}
The project documents the research carried out on the traditional dishes of Xicotepec, because, although it has the name of Magic Town and this encourages tourism, little is the impact it has had for the gastronomic sector, being an attractive a unique gastronomy to those who are looking to know Mexico through its food. A cuisine that, due to the constant migration and globalization, it is being lost little by little, for this, economic entities and inhabitants of different places that prepare food (700 samples) were surveyed, with visits and practices with the traditional cookers of each community. Based on the analysis and interpretation of the information, favorable results were obtained with respect to the cuisine, its ingredients and ways of preparation, and an anecdotal recipe book was prepared in order to train the students of gastronomy of the Technological University of Xicotepec de Juarez, high school students with food training and students of the Centro de Capacitación y Desarrollo (CECADE), in order to promote culinary preservation and interest in local gastronomic research to achieve specialization and improve the preparation of typical dishes of the region in their future work centers.
\end{abstract}

Citación: GONZÁLEZ DE LOS MONTERO-SIERRA, María José, CRUZ-CABRERA, Clotilde, VERGARA-HERRERA, Francisco Javier y CARMONA-GONZÁLEZ, Juan Carlos. Preservación de tradiciones en el ámbito culinario para fortalecer la sustentabilidad turística en Xicotepec mediante la capacitación académica. Revista de Didáctica Práctica. 2019. 3-9: 8-13.

*Correspondencia al Autor (Correo Electrónico: mariajose.gonzalez@utxicotepec.edu.mx)

$\dagger$ Investigador contribuyendo como primer autor. 


\section{Introducción}

Los retos en materia educativa a nivel Superior son cada vez más demandados, debido a la necesidad colectiva de profesionistas especializados en diferentes ramas que aporten conocimientos y habilidades en el desarrollo social y económico en la región.

Los restaurantes, como cualquier empresa, requieren de especialistas integrales que apliquen sus conocimientos en pro del desarrollo de la entidad; necesidad cubierta por instituciones de capacitación para el trabajo, como CECADE e ICATEP, así como instituciones públicas de Educación Media Superior que imparten en sus contenidos académicos las capacitaciones y/o carreras técnicas en alimentos y bebidas o turismo, como es el caso de los Colegios de Bachilleres y los CBTis.

Por otro lado, a Nivel Superior, encontramos licenciaturas profesionales y a nivel Técnico Superior Universitario, ofertadas en la Sierra Norte por Universidades privadas y públicas, como lo es la Universidad Tecnológica de Xicotepec de Juárez.

Dichas instituciones tienen la obligación, el valor social y ético de preparar a jóvenes competentes para realizar actividades encomendadas por las empresas, así como también le brindan al estudiante la capacidad de adaptarse a diversos contextos; con el fin de aportar sus servicios y cubrir las necesidades y cumplimiento de las metas organizacionales.

\section{Justificación}

Dentro del sector restaurantero se apertura áreas de oportunidad que se enfocan en gran medida al sector gastroturístico.

El turismo en la región ha sido incentivado por el programa de pueblos mágicos, al que Xicotepec pertenece desde el año 2012. Sin embargo, debido al difícil acceso a las distintas comunidades, rancherías y juntas auxiliares que conforman la totalidad del municipio, el turismo se ubica estancado únicamente en la cabecera municipal, por lo cual el visitante difícilmente conoce a fondo las tradiciones y los atractivos del lugar.

\section{Problema}

Hoy en día, la cultura gastronómica tradicional de los pueblos mexicanos, se pierde gradualmente debido a la migración de los habitantes, la globalización y la falta de interés por parte de las nuevas generaciones de preservar su cultura e identidad. Es debido a ello que, incluso los oriundos de Xicotepec pertenecientes a generaciones jóvenes y de mediana edad, desconocen por completo muchos de los platillos tradicionales característicos de la región.

\section{Hipótesis}

Mediante la capacitación de los estudiantes en la Sierra Norte del estado de Puebla, se podrá preservar la cultura culinaria con la implementación de un recetario anecdótico, resultado de una investigación en las diferentes juntas auxiliares de Xicotepec de Juárez, lo que servirá como estrategia en el fomento al turismo gastronómico.

\section{Objetivos}

\section{Objetivo General}

Crear estrategias para preservar la cocina endémica de Xicotepec pueblo mágico y promocionarla como turismo gastronómico, mediante la capacitación de estudiantes de la UTXJ y otros centros de capacitación utilizando un recetario anecdótico.

\section{Objetivos específicos}

- Documentar información sobre la cocina endémica de Xicotepec mediante recetario anecdótico.

- Recabar información sobre los antecedentes de la comida endémica y sus respectivos ingredientes principales o más utilizados.

- Establecer estrategias de mejora para los negocios con capacitación en base a sus platillos y el servicio que ofrezcan, destacando la cocina endémica. 


\section{Marco teórico}

La cocina de mexicana es nombrada en el 2010 como patrimonio inmaterial de la humanidad por la Organización de las Naciones Unidas para la Educación, la Ciencia y la Cultura (UNESCO), siendo un acontecimiento único en la gastronomía mexicana como lo indica la revista Forbes life (2016) es por ello que mundialmente representa importancia en el ámbito culinario, hablar de la gastronomía que ofrece el País, es hablar de un sinfín de cultura; a través de estos productos de materia educativa se fortalece la identidad de los educandos que se forman en instituciones que brindan la especialización en la gastronómica, como las ya antes mencionadas, permitiéndoles replicar su conocimiento de lo aprendido en su formación reflejándose en las labores dentro del sector restaurantero.

De acuerdo con, personas mayores con experiencia y conocedores de cultura dicen: "Las cocinas endémicas son aquellas que proporcionan comida al cliente por un instintivos económico la cual es distinguida en por en el lugar ya que cuenta con una preparación tradicional, así como también los ingredientes".

Esta es basada en productos naturales únicos de una región y estacionales. Posee cualidades derivadas del lugar de producción extracción o cultivo, y que están sometidos a factores locales específicos como clima y el terreno.

\section{Metodología de la investigación}

La investigación se realizó de manera cuantitativa y cualitativa a través de una serie de preguntas a cocineras tradicionales y a empresarios de la industria gastronómica, con el objetivo de identificar los recursos que destaquen a Xicotepec y así disminuir la problemática claramente en el ámbito explicativo ya que va más allá de la descripción detallando los factores que intervienen en el consumo de la comida endémica

De la población encuestada se realizaron los cálculos siguientes, para determinar el análisis de la situación con mayor exactitud, de esta manera poder ejercer una posible solución y alternativas ante el estudio

\begin{tabular}{|l|l|r|}
\hline \multicolumn{3}{|c|}{ Cálculo del tamaño de una muestra } \\
\hline \multirow{2}{*}{$N^{*}\left(\alpha_{c}^{*} 0,5\right)^{2}$} \\
\hline $1+\left(e^{2 *}(N-1)\right)$ & Error & $3.7 \%$ \\
\cline { 2 - 3 } & Tamaño población & 7,030 \\
\cline { 2 - 3 } & Nivel de confianza & $96 \%$ \\
\cline { 2 - 3 } & Tamaño de la muestra $=$ & 703 \\
\hline
\end{tabular}

Tabla 1

La población total de aplicación de la encuesta son 703 personas, se tomó como margen de error $3.7 \%$ de la población total de cada comunidad la cual se presenta graficado según la comunidad el porcentaje de población a representar con el margen utilizado.

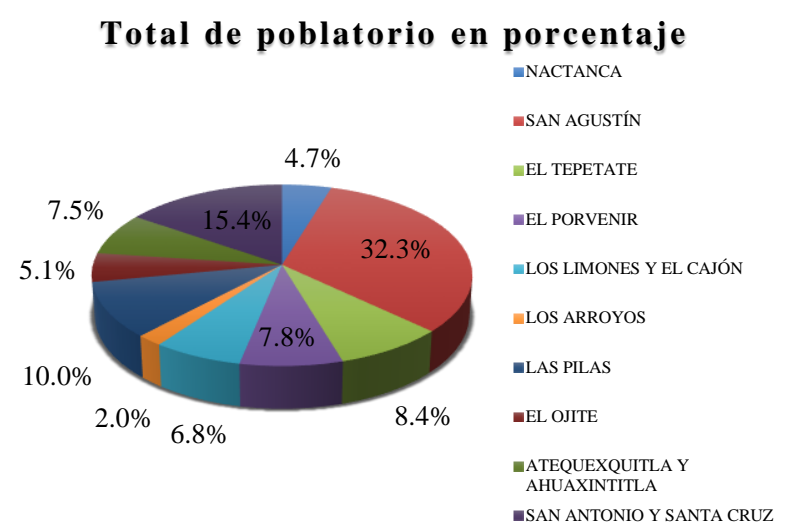

Gráfico 1

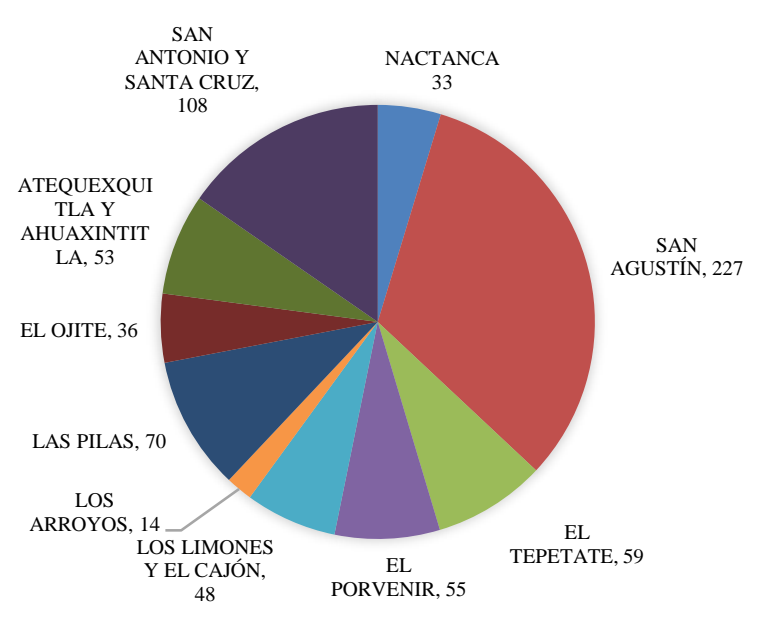

Gráfico 2

\section{Resultados}

La presente investigación abarco diez comunidades pertenecientes al municipio de Xicotepec de Juárez, todas ellas enclavadas en la Sierra Norte de Puebla, por lo que su aislamiento natural ha limitado la difusión de sus atractivos turísticos y platillos destacados, así como sus ingredientes. 
Las siguientes comunidades que fueron seleccionadas para su encuestar a su población son: Nactanca (chica, grande y ejido), San Agustín, El Tepetate, El Porvenir, Los Limones y el Cajón, Los Arroyos, Las Pilas, El Ojite, Atequexquitla y Ahuaxintitla, San Antonio y Santa Cruz. De acuerdo a la recopilación de datos y registros anecdóticos cualitativos se pudo obtener la compilación de ingredientes y recetas característicos propias de cada pueblo, permitiendo a su vez la elaboración y estandarización de un recetario que compila los platillos más destacados de cada comunidad.

Mediante el recetario elaborado, se especializa al alumnado del área de gastronomía de la Universidad Tecnológica de Xicotepec de Juárez, así como a los prestadores de servicios y egresados de centros de capacitación, en la producción y montaje de dichos platillos, como estrategia turística para las sociedades ajenas al municipio de Xicotepec de Juárez, ofertando más bagaje gastronómico al que ahora se oferta en la cabecera municipal.

Con ello se propone a los empresarios restauranteros que utilicen dicha estrategia para ofertar los productos o platillos regionales que se obtuvieron a partir de la investigación preservando el patrimonio culinario de la región y permitiendo al turista conocer las costumbres $\mathrm{y}$ tradiciones culinarias reales del municipio. Se muestran los siguientes resultados:
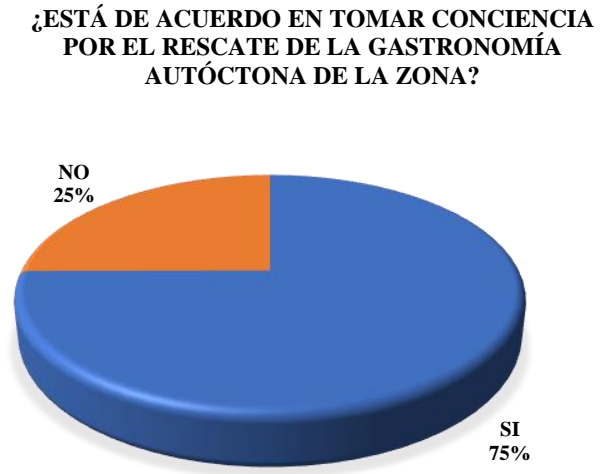

Gráfico 3

¿ESTARÍA DISPUESTO A COMPARTIR PARTE DE SU CULTURA CON OTRAS COMUNIDADES DE LA ZONA?

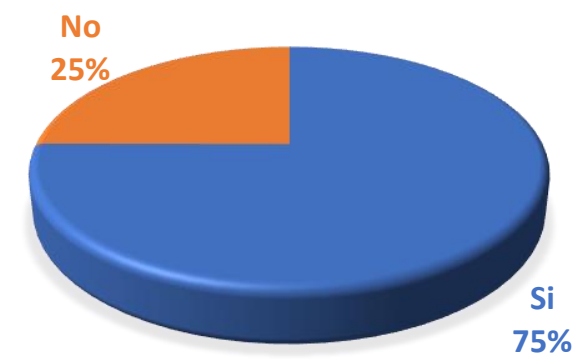

Gráfico 4

ISSN: 2523-2444

ECORFAN ${ }^{\circledR}$ Todos los derechos reservados

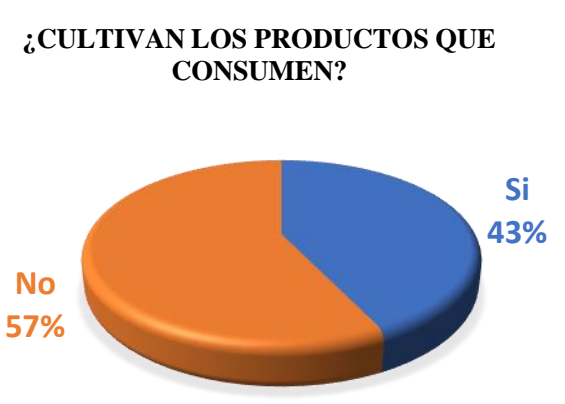

Gráfico 5

¿TIENE CONOCIMIENTO SOBRE LA CULTURA DE ESTE LUGAR?

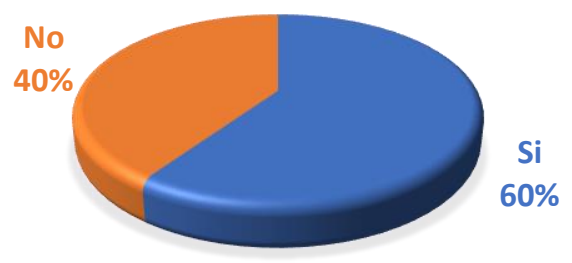

Gráfico 6

¿TODOS LOS ALIMENTOS QUE UTILIZAN EN
LA PREPARACIÓN DE SU COMIDA LAS CONSIGUEN EN LA LOCALIDAD?

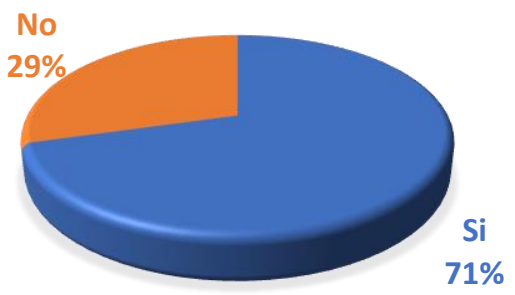

Gráfico 7

¿CREE QUE ES SUFICIENTE EL INTERÉS DEL PUEBLO PARA DAR A CONOCER SU GASTRONOMÍA?

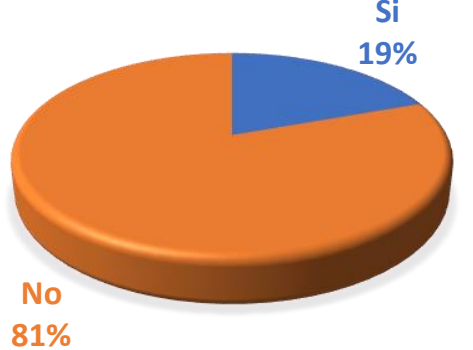

Gráfico 8

CREES QUE EL PROYECTO QUE SE PRETENDE REALIZAR ES BUENO PARA RESCATAR LOS PLATILLOS DE LA LOCALIDAD?

PLATILLOS
No

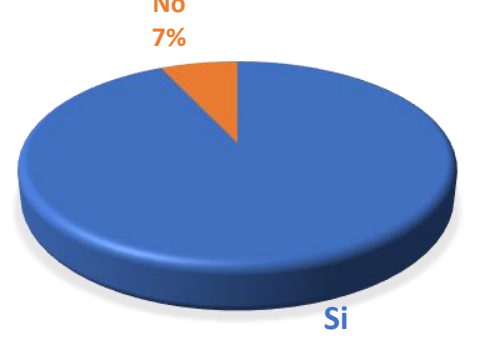

$93 \%$

Gráfico 9

GONZÁLEZ DE LOS MONTERO-SIERRA, María José, CRUZ-CABRERA, Clotilde, VERGARA-HERRERA, Francisco Javier y CARMONA-GONZÁLEZ, Juan Carlos. Preservación de tradiciones en el ámbito culinario para fortalecer la Juan Carlos. Preservación de tradiciones en el ámbito culing pa Revista de Didáctica Práctica. 2019 


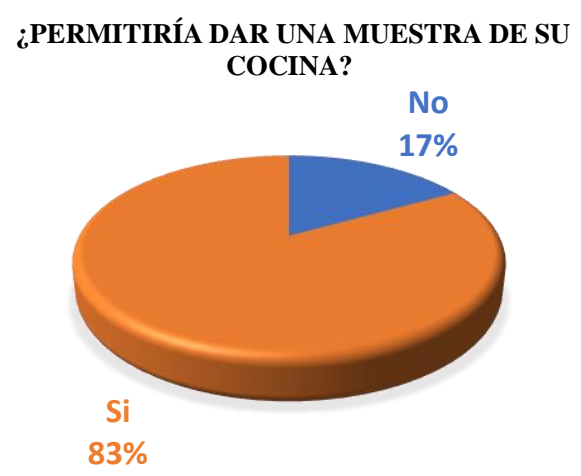

Gráfico 10

\section{Análisis de los resultados para la obtención de beneficios de la preservación de la cultura gastronómica}

La recopilación de recetas y su aplicación en la formación académica de los futuros prestadores de servicios culinarios es de amplio impacto para Xicotepec, aportando beneficios sociales, culturales, económicos y profesionales, que contribuyan con la gastronomía que se tiene en el municipio; así como lo menciona la Secretaría de Turismo que es necesario mostrar la diversidad del arte popular mexicano en los distintos ámbitos culturales, destacando sabores $\mathrm{y}$ conocimientos de creadores y portadores, procesos elaboración y mecanismos de protección y continuidad para las siguientes generaciones (Conaculta, 2017) ${ }^{1}$, por ello ante lo mencionado es importante registrar recetas únicas o características de las comunidades para los colegiados que estudien o se profesionalicen en el arte culinario vayan disminuyendo las posibilidades de que queden olvidadas.

\section{Siendo los beneficios los siguientes:}

El rescate de ingredientes, métodos y platillos típicos de las distintas juntas auxiliares, mismos que se van perdiendo debido a factores externos, generalmente económicos y sociales, así como a la llegada de productos variados a los cuales la población puede acceder con mayor facilidad, tanto en desgaste físico como económico, pues muchos de los ingredientes endémicos han ido escaseando por el cambio climático y su obtención agrícola requiere mayor esfuerzo, razón por la cual se encarece para su adquisición en los mercados locales o en la plaza municipal.
La preservación de la identidad de las comunidades y el municipio. Una vez rescatados de perderse en el tiempo y la globalización, la elaboración del recetario genera una evidencia escrita que registra para la sociedad en general la existencia de dichos insumos y sus métodos de cultivo o recolección, así como de los platillos realizados con los mismos y los procedimientos típicos para su elaboración.

El fomento al turismo gastronómico en la región. Si bien el nombramiento de Pueblo Mágico, la privilegiada ubicación, sus atractivos y sus recursos naturales hacen de Xicotepec un lugar turístico por sí mismo, la oferta de alimentos y bebidas es también clave para la atracción de visitantes. La nueva visión gastronómica de volver a los orígenes haciendo uso de ingredientes y técnicas locales en la creación de platillos ha dado lugar a un turismo enfocado en los atractivos culinarios de los destinos turísticos. Bajo esta visión, los visitantes más jóvenes ya no visitan estas plazas sólo por sus paisajes o atractivos naturales o arquitectónicos, sino también gastronómicos, buscando no únicamente conocer los edificios, panoramas e historias del lugar que visitan, sino también conocer las poblaciones por su comida, ingredientes y cultura culinaria. El incluir en las cartas de los restaurantes locales platillos endémicos de la región se complace al visitante, mejorando su experiencia y la impresión que se lleva del lugar.

El crecimiento del movimiento económico local. La oferta de platillos tradicionales que ocupen ingredientes locales incentivará el flujo económico, debido en primer lugar a los ingresos generados por la derrama económica que los turistas dejan tras su consumo. Por otra parte, los productores locales se ven beneficiados al colocar sus productos con mayor facilidad, sin intermediarios y recibiendo directamente el efectivo fruto de sus labores agrícolas.

El valor agregado en la formación profesional de los estudiantes de cualquier nivel enfocados al sector gastroturístico, quienes tendrán una especialización en la cocina regional, sumado a una mayor cultura culinaria.

\footnotetext{
${ }^{1}$ (Consejo Nacional para la Cultura y las Artes, 2017) 
La apertura para la formación de una ruta gastronómica que sirva como eje turístico en el que los turistas observarán de primera mano las actividades de producción de ingredientes, así como la elaboración de los platillos de mano de las cocineras tradicionales, haciendo uso de los métodos ancestrales característicos. La cultura culinaria descubierta en las comunidades y juntas auxiliares de Xicotepec de Juárez Dentro del trabajo de campo realizado para la recopilación de las recetas para integrar el registro, se llevaron a cabo prácticas con cocineras tradicionales de cada comunidad con el fin de conocer de primera mano los métodos tradicionales de preparación. Las recetas encontradas en cada junta auxiliar fueron las siguientes:

En Nactanca (chica, grande y ejido) se obtuvo la receta de: "los canaxtles"; en San Agustín Atlihuacán: "las gorditas de frijol con salsa molcajeteada y pipián con acuyo y atole de nixtamal con hoja de naranjo"; la comunidad del Tepetate con su "huatape y el uso de la hoja de pimienta", en la junta auxiliar del Porvenir con: "el tamalito de pascal de chayote"; en Los Limones y el Cajón con la receta del "revoltijo"; Los Arroyos con su platillo de "tamales de pipián y chayote acompañado de un atole de calabaza de castilla"; en la comunidad de Las Pilas se obtiene "el adobo con cancelil"; en la ranchería del Ojite se obtuvo la receta del "panili" y mientras en Atequexquitla y Ahuaxintitla se preservo la receta del "pipián de chota" y por último, en San Antonio Ocopetlán y Santa Cruz coincidente "los tamales de chayote", pero con un diferente procedimiento.

Las mujeres reconocidas por su sazón, muchas de ellas entradas en años y cuyas técnicas les fueron legadas por sus madres y abuelas, siguiendo fielmente la tradición oral, abrieron sus puertas de sus hogares y permitieron que los especialistas culinarios observaran el desarrollo de las preparaciones, ayudando también en el ámbito práctico con el préstamo de sus cocinas de humo, esencia de la cocina mexicana, para replicar fielmente las preparaciones características de sus comunidades. El apoyo de estas mujeres de humo, así como de los campesinos locales es fundamental para conservar registros fieles más allá de la palabra, manteniendo a salvo el patrimonio inmaterial de la región.

\section{Conclusiones}

La permanencia y conocimiento de costumbres culinarias en las nuevas generaciones es un reto para la sociedad actual, como se mostró en el análisis de las encuestas, debido a que la demanda y cambios sociales, hacen que las cocineras de tradición se enfoquen en una vida laboral dejando atrás estas prácticas culturales gastronómicas que se elaboraban en casa. La información obtenida propuesta en un recetario de la región, generado por la Universidad Tecnológica de Xicotepec de Juárez permite contribuir con la preservación de las tradiciones culinarias y a su vez son una estrategia para la atracción turística debido a la oferta de experiencias gastronómicas únicas y propias del municipio de Xicotepec.

El valor agregado que obtendremos de la estrategia por la cual se está optando, es dar por medio de la identidad gastronómica del Pueblo Mágico de Xicotepec, la exclusividad de recetas no muy conocidas debido a que son propias de las comunidades a las cuales se fueron encuestadas. Es por eso que se ve la importancia de estudiar e investigar a fondo la gastronomía, su entorno social y cultural, y resaltar así, la gastronomía que tiene este lugar, pues se ha ido viendo a través del tiempo, que se van perdiendo por falta de interés o por una vida más práctica, los platillos más típicos y tan arraigados al lugar, tanto que turistas que lo visitan, se van con la idea que su gastronomía se define en unos cuantos platillos, siendo en realidad, que es una fuente rica en materiales naturales con su rica gastronomía.

\section{Referencias}

Consejo Nacional para la Cultura y las Artes. (04 de Mayo, de 2017). Obtenido de www.conaculta.gob.mx

Fernández, E. (2016). Cómo llegó la gastronomía mexicana a ser patrimonio de la humanidad. Septiembre 16, 2019, de Forbes Life. Sitio web: https://www.forbes.com.mx/forbeslife/gastronomia-mexicana-patrimonio-de-lahumanidad/ 